\title{
Unexpected hardening effects in bilayered gel beams
}

\author{
Paola Nardinocchi · Eric Puntel
}

Received: date / Accepted: date

\begin{abstract}
A classical problem in structural mechanics is the evaluation of beam stretching and curvature in slender bilayered beams, due to mechanical actions, thermal distortions, differential growth, and more recently, to swelling. We investigate the non-monotonic changes in the curvature of swollen bilayer beams due to mismatches in physical properties of the two layers starting from a simple structural approach, and discuss the apparent contrast with the well-known Timoshenko's formula through a scaling analysis. Due to the large strains involved in the problem, we also discuss the problem through a thermodynamics based on Gent model for the elastic contribution to the free-energy of the gels.
\end{abstract}

Keywords swelling - geometric composites · bilayer beams

PACS 46.05.+b - 81.05.Qk

\section{Introduction}

A classical problem in structural mechanics is the evaluation of beam stretching and curvature in slender bilayer beams, due to mechanical actions, thermal distortions, differential growth, and more recently, to swelling (???). The design principle of bending actuation using

\section{P. Nardinocchi}

Dipartimento di Ingegneria Strutturale e Geotecnica, Sapienza Università di Roma, via Eudossiana 18, 00184 ROMA

Tel.: +39-06-44585242

Fax: +123-45-678910

E-mail: paola.nardinocchi@uniroma1.it

E. Puntel

Dipartimento Politecnico di Ingegneria e Architettura, Universit di Udine, via del Cotonificio 114, Udine I-33100, Italy a layered structure dates back to the pioneering work of Timoshenko (?). Therein, the curvature of a bimetal strip induced by thermoelastic deformations was derived as proportional to the difference in elongation of the two layers and inversely proportional to the thickness of the strip. Following that formula, fixed the ratio $\beta$ of the thickness of the top layer with respect to the total thickness of the beam and the ratio $\alpha$ of the two elastic moduli, beam curvature depends on the difference in linear swelling ratios, which increases for pairs of softer layers sharing the same elastic moduli's ratio $\alpha$. It is worth noting that, recently, Timoshenko's analysis was borrowed for a qualitative estimation of the curvature of hydrogel systems under swelling (??).

However, in bilayer gel beams things are different from bimetal strips, mainly due to the huge deformations involved. To the best of our knowledge, the first class of environmentally responsive structures based on the spatial modulation of the chemical nature of gels was proposed in (?). Therein, the modulation was achieved by limiting the interpenetration of part of one gel network with another gel network. The gels so produced had an internally modulated structure: the spatial modulation of the chemical nature of gels determined a spatial modulation of the mechanical characteristics and realized a bilayer gel beam. In particular, the bending of the beam was obtained by varying both temperature and acetone concentration of the bath, as each of the two layers drastically shrank in response to only one of the two stimuli. In (?), the extreme bending of a bilayer hydrogel beam was proposed as a tool to control the direction and kinetics of molecular release. The bilayer beam bent and self-folded forming a hydrogel tube; this self-folding property was attained by forming double-layered hydrogel patches with significantly different swelling ratios (and elastic moduli). The diam- 
eter of the gel tubes could be predicted by an equation used to estimate the curvature of the beam. The finite bending of a bilayer gel in response to temperature under plane strain condition was also studied in (?). They predicted larger bending curvatures of bilayer beams compared with the linear model based on the elementary beam theory, and showed as a high swelling ratio in the bilayer enhances the differences between both models. Deformation modes in bilayered soft active structures have been recently studying through modern techniques based on Gamma-convergence, with the aim to show, also in the context of soft active mecanics, the consistency of the structural reduced models for plates (?).

We started to investigate swelling-induced bending in bilayer gel beams in (?) through an approximated uncoupled theory of stress-diffusion, which was also reformulated in (?) within the context of a finite and exact bending theory. Here, we investigate both analytically and numerically the non-monotonic changes in the curvature of swollen bilayer beams due to mismatches in physical properties of the two layers starting from the approach proposed in (?). In particular these changes occur if the stiffness of the two layers is decreased while keeping their ratio $\alpha$ fixed or, similarly, if the free swelling of the layers in undisturbed conditions is increased without modifying $\alpha$. The apparent contrast with the well-known Timoshenko's formula is explicitly discussed through a scaling analysis which highlights as, neglecting that non-monotonic trend, recently also analyzed in (?), may lead to large overestimation of beam curvature. Due to the large strains involved in the problem, we also discuss the problem through a thermodynamics based on Gent model for the elastic contribution to the free-energy of the gels (??). As expected, for any free-swelling stretches Gent's model provides a curvature which is always smaller than the neo-Hookean model and, what is more important within this context, decreases when the bilayer becomes softer and softer.

\section{Bilayer beams under growth-induced plane bending}

We consider a beam, made by the assembly of two different beam layers of rectangular cross section. We assume that the top (t) beam has a Young's modulus $Y_{t}$ smaller than the Young's modulus $Y_{b}$ of the bottom (b) beam and set $\alpha=Y_{t} / Y_{b} \leq 1$. We also assume that the beam has total thickness $h=h_{t}+h_{b}$, and set $\beta=h_{t} / h$, being $h_{t}$ and $h_{b}$ the thicknesses of the homogeneous top and bottom beam-like parts, respectively: $\beta=0$ when $h=h_{b}$ and $\beta=1$ when $h=h_{t}$. The length and the width of the beam are denoted as $l$ and $b$, respectively; we further postulate $h / b>1$.

In (?), it has been shown that the deformative process induced by a differential growth within the two layers may be analysed within the context of beamunder-bending theory. Therein, the longitudinal stretch $\lambda$ of the bilayer beam was characterised in the form

$\lambda\left(x_{3}\right)=\Lambda_{0}\left(1+x_{3} \Lambda_{0} \kappa\right)$,

in terms of the uniform, possibly large, longitudinal stretch $\Lambda_{0}$ and the uniform curvature $\kappa$ of the beam axis $(\kappa>0),-h / 2 \leq x_{3} \leq h / 2$ being the thickness coordinate in the dry configuration. Assuming that: (i) the top and bottom homogeneous beam-like layers suffer growth (longitudinal) deformations $\lambda_{o t}$ and $\lambda_{o b}$ and that further elastic (longitudinal) deformations $\lambda_{e t}$ and $\lambda_{e b}$ arise to recover the structural integrity of the beam, in such a way that the following decompositions hold

$\lambda_{e t}=\lambda \lambda_{o t}^{-1} \quad$ and $\quad \lambda_{e b}=\lambda \lambda_{o b}^{-1} ;$

(ii) out-of-plane stresses are zero and the corresponding longitudinal stresses $\sigma_{t}$ and $\sigma_{b}$ on the cross-sections of the top and bottom layers, can be evaluated as

$\sigma_{t}\left(x_{3}\right)=Y_{t}\left(\lambda\left(x_{3}\right) \lambda_{\text {ot }}^{-1}-1\right),(h / 2-\beta h)<x_{3}<h / 2$

$\sigma_{b}\left(x_{3}\right)=Y_{b}\left(\lambda\left(x_{3}\right) \lambda_{o b}^{-1}-1\right),-h / 2<x_{3}<(h / 2-\beta h) ;$ the resultant force $F$ and torque $M$ of the longitudinal stresses are determined in terms of $\Lambda_{o}$ and $\kappa$ from the following equations:

$$
\frac{F}{b}=\lambda_{o b}^{2} \int_{-h / 2}^{h / 2-\beta h} \sigma_{b}\left(x_{3}\right) d x_{3}+\lambda_{o t}^{2} \int_{h / 2-\beta h}^{h / 2} \sigma_{t}\left(x_{3}\right) d x_{3}
$$

$\frac{M}{b}=\lambda_{o b}^{2} \int_{-h / 2}^{h / 2-\beta h} x_{3} \sigma_{b}\left(x_{3}\right) d x_{3}+\lambda_{o t}^{2} \int_{h / 2-\beta h}^{h / 2} x_{3} \sigma_{t}\left(x_{3}\right) d x_{3}$.

If no external forces are applied, under unconstrained conditions,

$F=\hat{F}\left(\Lambda_{0}, \kappa\right)=0 \quad$ and $\quad M=\hat{M}\left(\Lambda_{0}, \kappa\right)=0$.

In order to discuss the dependence of beam stretch $\Lambda_{0}$ and curvature $\kappa$ on the growth deformations $\lambda_{o b}$ and $\lambda_{o t}=\lambda_{o b} / \Gamma$, we introduce non-dimensional variables $X_{0}$ and $X_{1}$ as follows:

$\Lambda_{0}=X_{0} \lambda_{o b} \quad$ and $\quad \kappa h=X_{1} X_{0}^{-2} \lambda_{o b}^{-1}$.

With this (??) become:

$\lambda \lambda_{o t}^{-1}=\Gamma\left(X_{0}+s_{3} X_{1}\right)$ and $\lambda \lambda_{o b}^{-1}=X_{0}+s_{3} X_{1}$,

where $s_{3}$ is a non-dimensional coordinate equal to $x_{3} / h$. Substituting (??) into (??), equations (??) and (??) deliver a linear system of equations in $X_{0}$ and $X_{1}$ whose 
coefficients depend solely on $\alpha, \beta$ and $\Gamma$. Looking at (??) this means that whenever the ratios $\alpha$ and $\beta$ are held constant while growth deformations $\lambda_{o b}$ and $\lambda_{o t}$ are increased keeping their ratio $\Gamma$ also constant, the curvature of the beam decreases and is inversely proportional to the growth deformation of the bottom layer $\lambda_{o b}$. This result is in contrast with Timoshenko's result at small strains (?). On the other hand, the fact that curvature $\kappa$ is inversely proportional to the thickness of the beam keeps holding at finite strains. The solution of the equations (??)-(??) yields a representation of $X_{0}$ and $X_{1}$ solely depending on $\alpha, \beta$, and $\Gamma$ :

$$
\begin{aligned}
X_{0} & =1+\frac{1}{\Gamma}\left(\alpha \beta(1-\Gamma)\left(\alpha \beta^{3}+\left(1-\beta^{3}\right) \Gamma\right)\right) . \\
& \cdot\left(\alpha^{2} \beta^{4}+2 \alpha \beta\left(\left(1-\beta^{2}\right)+(1-\beta)^{3}\right) \Gamma+\right. \\
& \left.+(1-\beta)^{4} \Gamma^{2}\right)^{-1}, \\
X_{1} & =6 \alpha(1-\beta) \beta(1-\Gamma) . \\
& \cdot\left(\alpha^{2} \beta^{4}+2 \alpha \beta\left(\left(1-\beta^{2}\right)+(1-\beta)^{3}\right)+\right. \\
& \left.+(1-\beta)^{4} \Gamma^{2}\right)^{-1} .
\end{aligned}
$$

\section{Swelling-induced growth}

Swelling-induced growth in gel-based actuators depends on the solvent distribution inside the gel and on the rate of solvent uptake at the boundary. Unhampered by either loads or restraints, swelling induced by a solvent yields changes in size that manifest as isotropic changes once a steady state is reached. Here, we refer to the nonlinear three-dimensional stress-diffusion model presented in (?), revised in (?), and already used in (?) to discuss the role of equation (??) in swellinginduced growth. Shortly, that model is based on a coupled system of equations which describe: (i) the balance of stress $\mathbf{S}_{d}\left(\left[\mathbf{S}_{d}\right]=\mathrm{J} / \mathrm{m}^{3}\right)$ and of solvent concentration $c_{d}\left(\left[c_{d}\right]=\mathrm{mol} / \mathrm{m}^{3}\right)$

$\operatorname{div} \mathbf{S}_{d}=\mathbf{0}, \dot{c}_{d}=-\operatorname{div} \mathbf{h}$,

$\mathbf{h}\left([\mathbf{h}]=\mathrm{mol} / \mathrm{m}^{2} \mathrm{~s}\right)$ being the solvent flux and subscript $d$ denoting the dry configuration, holding at any time on the three-dimensional region $\mathcal{B}_{d}$ of the Euclidean space $\mathcal{E}$ which identifies the dry reference configuration of the bilayer beam; (ii) the boundary conditions for traction t or displacement $\overline{\mathbf{u}}$

$\mathbf{S}_{d} \mathbf{m}=\mathbf{t}, \quad$ and $\quad \mathbf{u}=\overline{\mathbf{u}}$,

and for solvent flux $q$ or chemical potential $\mu_{e}$

$-\mathbf{h} \cdot \mathbf{m}=q$ and $\quad \mu=\mu_{e}$, which may be posed on the appropriate parts of the boundary at any time (depending on the problem under study); (iii) the initial conditions $\left(\mathbf{u}_{o}, c_{d o}\right)$ for the state variables $\mathbf{u}$ and $c_{d}$ of the model; (iv) the kinematic constraint enforcing that change in volume is only due to solvent uptake or release, that is, both the polymer and the solvent are assumed to be incompressible and the actual volume-element $d v$ is the sum of the dry volume-element $d V_{d}$, plus the solvent volume-element $d V_{\text {sol }}=\Omega c_{d} d V_{d}$ :

$d v=J_{d} d V_{d}=d V_{d}+d V_{\text {sol }}=\left(1+\Omega c_{d}\right) d V_{d}$,

hence, $J_{d}=\operatorname{det} \mathbf{F}_{d}=1+\Omega c_{d}$ with $\mathbf{F}_{d}=\mathbf{I}+\nabla \mathbf{u}$ and $\Omega$ the solvent molar volume; (v) the constitutive equations for the stress $\mathbf{S}_{d}$, the chemical potential $\mu([\mu]=\mathrm{J} / \mathrm{mol})$ and the flux $\mathbf{h}$ which yield a further coupling between mechanics and chemistry of the gel:

$\mathbf{S}=\mathbf{S}_{e}\left(\mathbf{F}_{d}\right)-p \mathbf{F}_{d}^{*}, \quad \mu=\mu\left(c_{d}\right)+p \Omega$,

with $\mathbf{S}_{e}\left(\mathbf{F}_{d}\right)=\partial \psi / \partial \mathbf{F}_{d}, \mu\left(c_{d}\right)=\partial \psi / \partial c_{d}$, and the indeterminate pressure field $p$, coupling stress and chemical potential fields, interpreted as the reaction associated to the volumetric constraint. The free-energy $\psi$ describes the thermodynamics of the gel body, and the constitutive equation for the solvent flux $\mathbf{h}$ has to satisfy the reduced dissipation inequality (?):

$\mathbf{h} \cdot \nabla \mu \leq 0$.

As in (?), we assume that swelling in each component of the bilayer gel beam is realized by embedding it into a solvent bath, that it is unhampered by either loads or restraints, and only yields changes in size that manifest as isotropic changes once a steady state is reached. The steady state is characterised by zero stress and a chemical potential which is homogeneous within the body and equal to $\mu_{e}: \mathbf{S}_{d}=\mathbf{0}$ and $\mu=\mu_{e}$. When the free-energy of the gels is assumed in the Flory-Rehner form (??) and $\lambda_{o s}$ measures the isotropic swelling stretch induced by solvent uptake, the dry-reference stress $\mathbf{S}_{d}$ and the chemical potential $\mu$ of the gel, within each of the two gel layers (which we imagine to swell independently from one another) can be expressed as

$$
\begin{aligned}
\mathbf{S}_{d} & =\sigma \mathbf{I}, \quad \sigma=\frac{1}{3} Y_{s} \lambda_{o s}-\lambda_{o s}^{2} p \\
\mu & =\mathcal{R} T\left(\log \left(1-\frac{1}{\lambda_{o s}^{3}}\right)+\frac{1}{\lambda_{o s}^{3}}+\frac{\chi}{\lambda_{o s}^{6}}\right)+p \Omega,
\end{aligned}
$$

$s=t, b$ being a dummy indicator for the top or bottom layer, $Y_{s}$ the Young's modulus, $\mathcal{R}$ the gas constant and $T$ the temperature. ${ }^{1}$ With this, the free-swelling bath

1 Actually, the representation form of the stress $\sigma$ depends on the above cited incompressibility of the polymer as $\sigma=$ $G_{s} \lambda_{o s}-\lambda_{o s}^{2} p$ with $G_{s}=Y_{s} /(2(1+\nu))$ and assuming that for incompressible material $\nu=1 / 2$ we get $G_{s}=Y_{s} / 3$. 
conditions deliver the following thermodynamic equilibrium equation for each gel layer:

$0=\frac{Y_{s} \Omega}{3 \lambda_{o s}}+\mathcal{R} T\left(\log \left(1-\frac{1}{\lambda_{o s}^{3}}\right)+\frac{1}{\lambda_{o s}^{3}}+\frac{\chi}{\lambda_{o s}^{6}}\right)$.

We consider the Flory-parameter $\chi$ as fixed and view the equation (??) as a nonlinear relation between swelling stretch $\lambda_{o s}$ and Young's modulus $Y_{s}$ of each layer: $Y_{s}=\hat{Y}_{s}\left(\lambda_{o s}\right)$. Figure ?? shows the $\hat{Y}_{s}$ relation, for $\Omega=6.023 \cdot 10^{-5} \mathrm{~m}^{3} / \mathrm{mol}, T=293 \mathrm{~K}$, and $\chi=0.2$ (solid blue line).

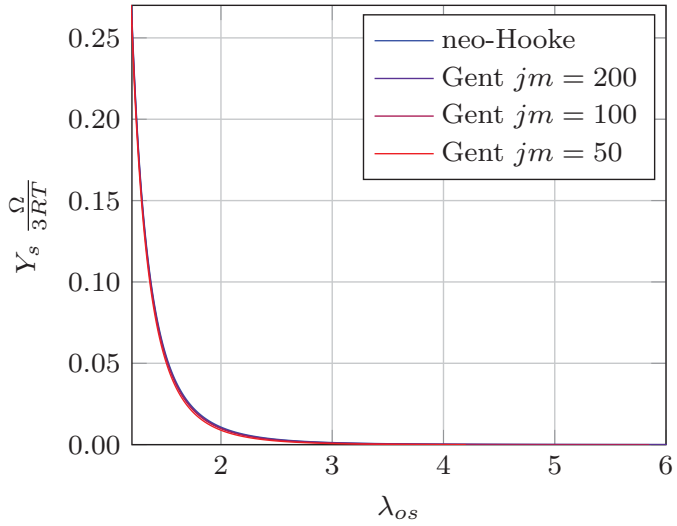

Figure 1 Young's modulus $Y_{s}$ versus free-swelling ratio $\lambda_{o s}$ dictated by equation (??) (solid blue line) and by equation (??) for $j_{m}=50,100,200$ (solid red lines). The corresponding solid lines are almost indistinguishable but the latter three reach zero stiffness for a finite value of stretch $\sqrt{j_{m} / 3+1}$.

With this, we get

$\alpha=\frac{Y_{t}}{Y_{b}}=\left(\frac{\lambda_{o b}}{\lambda_{o t}}\right)^{5} \frac{f\left(\lambda_{o t}\right)}{f\left(\lambda_{o b}\right)}=\Gamma^{5} \frac{f\left(\lambda_{o t}\right)}{f\left(\lambda_{o b}\right)}$,

with

$f\left(\lambda_{o s}\right)=\chi+\lambda_{o s}^{3}+\lambda_{o s}^{6} \log \left(1-\frac{1}{\lambda_{o s}^{3}}\right)$.

In gels, parameters $\alpha$ and $\Gamma$ are therefore not independent and both the longitudinal stretch $\Lambda_{o}$ and the curvature $\kappa$ of the beam axis may then be represented as functions of only three parameters, such as e.g. $\alpha, \beta$ and $\lambda_{o b}$.

A simple solution is obtained when the chemical equilibrium equation (??) is replaced by its asymptotic version based on the assumption that the free swelling stretch $\lambda_{o s}>>1$ (that is, $1 / \lambda_{o s}<<1$ ). The expression (??) can be approximated by estimating the leading order term in the asymptotic expansion up to $O\left(1 / \lambda_{s}^{3}\right)$, and we get:

$f\left(\lambda_{o s}\right) \simeq(\chi-1 / 2)$

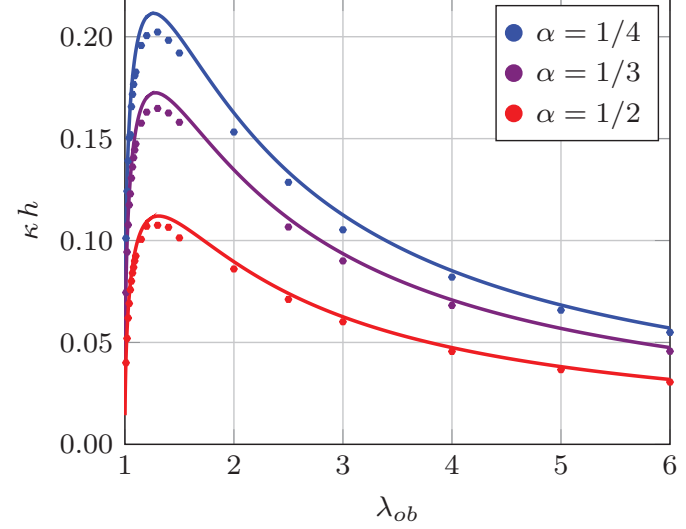

Figure 2 Curvature times thickness $\kappa h$ versus free-swelling stretch $\lambda_{o b}$ of the bottom layer for $\beta=1 / 2$ and $\alpha=$ $1 / 4,1 / 3,1 / 2$ as follows from equations (??) $)_{2}$ and (??) (solid lines) and from the computational model (coloured dots).

and

$\Gamma=\frac{\lambda_{o b}}{\lambda_{o t}}=\left(\frac{Y_{t}}{Y_{b}}\right)^{1 / 5}=\alpha^{1 / 5}$.

For large values of $\lambda_{o b}$, a frequent occurrence for gels, $\Gamma$ is independent of $\lambda_{o b}$. This has two consequences. Firstly, by substituting (??) into (??), we obtain expressions for $X_{0}$ and $X_{1}$ that depend only on $\alpha$ and $\beta$. Secondly and most importantly, equation (??) expresses the linear dependence of the stretch $\Lambda_{0}$ on $\lambda_{o b}$ and of the curvature $\kappa$ on $1 / \lambda_{o b}$ for fixed values of $\alpha$ and $\beta$. Then, the softer the two layers at constant $\alpha$, the larger the swelling stretches $\lambda_{o b}$ and $\lambda_{o t}$ and the smaller the curvature $\kappa$. In other words an unexpected hardening is observed. Such counterintuitive result disagrees with Timoshenko's result at small strains (?).

The two regimes, namely $\kappa \propto 1 / \lambda_{o b}$ for $\lambda_{o b}>>1$ and $\kappa$ growing with $\lambda_{o b}$ for $\lambda_{o b} \simeq 1$, are reconciled if the full expression (??) of $f\left(\lambda_{o s}\right)$ is used instead of its asymptotic expansion (??) to obtain the parameter $\Gamma$ to be used in (??). The plot in figure ?? shows $\kappa h$ vs. $\lambda_{o b}$ for $\alpha=1 / 4,1 / 3,1 / 2$ and $\beta=1 / 2$ (blue, purple, and red solid lines, respectively).

\subsection{Curvature slope singularity}

Figure ?? is suggesting that the slope of the non dimensional curvature $\kappa h$ vs $\lambda_{o b}$ plot may be vertical for $\lambda_{o b}$ close to 1 . In the following, we show that it is indeed so and explicitly determine the strength of the singularity. The obtained relationship should not be taken as a valid approximation of the $\kappa h$ vs $\lambda_{o b}$ relationship at small strains because numerical validations we performed showed only good asymptotic agreement that is, roughly, when $\lambda_{o b}-1<3.4 \times 10^{-4}$. However, care 
should be taken in adopting Timoshenko's linear $\kappa h$ vs $\lambda_{o b}$ relationship for gels at small strains, see eq. (??) in the following, as sometimes done in the literature.

We start from the nonlinear relationship (??) between Young's modulus $Y_{s}$ and free-swelling expansion $\lambda_{o s}$, with the aim to express the latter as a function of the former when $\lambda_{o} s$ is close to unity. Let us introduce the free-swelling strain $\varepsilon_{o} s$ and the dimensionless shear modulus $g_{s}$ as

$\lambda_{o s}=\varepsilon_{o s}+1 \quad$ and $\quad g_{s}=\frac{1}{3} \frac{Y_{s}}{R T / \Omega}$.

After approximating $\lambda_{o s}^{-n} \simeq 1-n \varepsilon_{o s}$, equation (??) becomes

$g_{s}\left(1-\varepsilon_{o s}\right)+\log \left(3 \varepsilon_{o s}\right)+\left(1-3 \varepsilon_{o s}\right)+\chi\left(1-6 \varepsilon_{o s}\right)=0$,

which yields the following crude approximation

$\lambda_{o s} \simeq \tilde{\lambda}_{o s}\left(g_{s}\right)=1+\frac{1}{3} \exp \left(g_{s}+\chi+1\right)$

Using equation (??), we can evaluate $\Gamma$ when $\lambda_{o b}$ is close to 1 . Given $\lambda_{o b}, g_{b}\left(\lambda_{o b}\right)$ is easily computed using (??) and (??); then, from $g_{t}=\alpha g_{b}$ the approximation $\lambda_{o t} \simeq \tilde{\lambda}_{o}\left(g_{t}\right)$ delivers:

$\Gamma=\frac{\lambda_{o b}}{\lambda_{o t}} \simeq \frac{\lambda_{o b}}{\tilde{\lambda}_{o}\left(\alpha g_{b}\right)}=\frac{\lambda_{o b}}{\tilde{\lambda}_{o}\left(-\alpha f\left(\lambda_{o b}\right) \lambda_{o b}^{-5}\right)}$.

Seeking a linear log-log relationship of the type $\log (1-$ $\Gamma) \simeq a \log \left(\lambda_{o b}-1\right)+b$ and skipping some lengthy passages one finds

$1-\Gamma \simeq \frac{3^{\alpha}}{3}\left(\lambda_{o b}-1\right)^{\alpha} \cdot e^{-(1-\alpha)(1+\chi)}$.

In the last step, the closed form expression of $\kappa h$ in terms of $\alpha, \beta, \Gamma$, obtainable from equations (??) and (??), is expanded to the first order around $\Gamma$ equal to 1 and yields:

$\kappa h \simeq \frac{6 \alpha \beta(1-\beta)}{(1-\alpha)\left((1-\beta)^{4}-\alpha \beta^{4}\right)+\alpha} \cdot(1-\Gamma)$.

Substituting (??) into (??) we get the asymptotic dependence of the curvature on $\lambda_{o b}$ and on the other model parameters $\alpha, \beta, \chi$. In particular, $\kappa h \propto\left(\lambda_{o b}-1\right)^{\alpha}$ which entails a vertical slope when $\lambda_{o b}$ is close to 1 since the ratio of Young's moduli $\alpha=Y_{t} / Y_{b}$ is assumed smaller than 1 in the present model.

\subsection{A constitutive model for huge deformations}

The local thermodynamic equilibrium equation (??) which relates Young's modulus and free-swelling stretch for each layer of the gel beam, strongly depends on the free energy chosen to describe the thermodynamics of swelling processes. We assumed that the Flory-Rehner free energy representation form holds, and split the free energy density $\psi$ into a Flory-Huggins mixing component $\psi_{m}$ and a neo-Hookean elastic component $\psi_{e}$. However, when the elastic stretches induced by swelling into the gel are very large, stiffening effects need to be considered, and one of the constitutive elastic models which accounts for the stiffening of polymer chains is the Gent model (??). So, we assumed unchanged the mixing component of the free-energy and represented the elastic component $\psi_{e}$ in the Gent form as:

$\psi_{e}\left(\mathbf{F}_{d}\right)=-\frac{1}{6} Y_{s} j_{m} \log \left(1-\frac{\mathbf{F}_{d} \cdot \mathbf{F}_{d}-3}{j_{m}}\right)$,

$j_{m}$ being a parameter which sets the ultimate deformation at which the network strands are fully stretched: in the limit $\mathbf{F}_{d} \cdot \mathbf{F}_{d}-3 \rightarrow j_{m}$, the Gent energy tends to $\infty$; for $j_{m} \rightarrow \infty$, the Gent model reduces to the neoHookean. Considering the isotropic swelling of the two layers delivered by $\lambda_{o s}(s=b, t)$, we have $\mathbf{F}_{d} \cdot \mathbf{F}_{d}=3 \lambda_{o s}^{2}$, and the ultimate deformation $\lambda_{o, m}$ is given by

$\lambda_{o, m}=\sqrt{j_{m} / 3+1}$.

The constitutively determined component $\mathbf{S}_{e}\left(\mathbf{F}_{d}\right)$ of the stress $\mathbf{S}_{d}$ corresponding to the Gent model is different from the neo-Hookean's and determines a change in the local thermodynamic equilibrium equation. Therefore, in Gent's model, (??) is replaced by:

$0=\frac{Y_{s} \Omega}{3 \lambda_{o s}} \frac{1}{1-\frac{3}{j_{m}}\left(\lambda_{o s}^{2}-1\right)}+\mathcal{R} T \frac{f\left(\lambda_{o s}\right)}{\lambda_{o s}^{6}}$,

being $f\left(\lambda_{o s}\right)$ defined as in (??) and $s=t, b$. We point out that, for the sake of simplicity, we are here with some loss of generality assuming that the value of $j_{m}$ is the same for the top and bottom layers. Equation (??) is still a nonlinear relation $\hat{Y}_{s}\left(\lambda_{o s}\right)$ between the free swelling stretch $\lambda_{o s}$ and Young's modulus $Y_{s}$ of each layer, parametrized by $j_{m}$. Once a value for $j_{m}$ is fixed, we can compare the Gent and neo-Hookean models in terms of the relation prescribed between $Y_{s}$ and $\lambda_{o s}$. In figure ??, we fixed $j_{m}=50,100,200$ and plotted that relation. The corresponding solid lines are almost indistinguishable from the one describing equation (??), though, differently from the neo-Hookean case, they reach zero stiffness for a finite stretch value $\sqrt{j_{m} / 3+1}$. It all depends on the choice of the parameter $j_{m}$ which sets the ultimate deformation at which the 


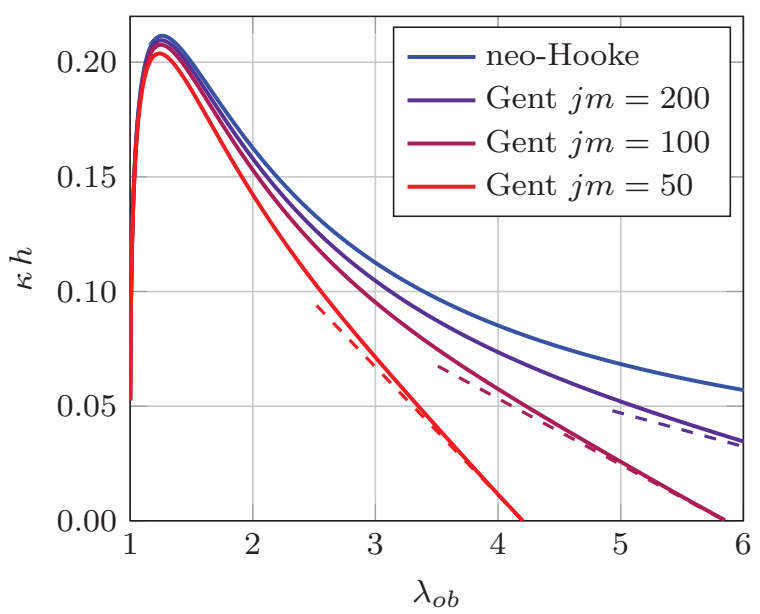

Figure 3 Curvature times thickness $\kappa h$ versus free-swelling stretch $\lambda_{o b}$ of the bottom layer for $\beta=1 / 2, \alpha=1 / 4$, and $j_{m}=50,100,200$. Dashed lines indicate the asymptotic trend (??).

network strands are fully stretched, that is, reducing $j_{m}$ the difference between the two constitutive description is amplified at smaller stretches. We selected our values from ?.

The block structure of our model allows us to easily evaluate the dimensionless curvature $\kappa h$ vs the free swelling ratio $\lambda_{o b}$ of the bottom layer based on an elastic Gent energy. Given $\alpha$ and $\lambda_{o b}$, it suffices to use (??) twice to first compute $Y_{b}\left(\lambda_{o b}\right)$ and then, given $Y_{t}=\alpha Y_{b}$, $\lambda_{o t}\left(Y_{t}\right)$; and, at the end, evaluate $\Gamma=\lambda_{o b} / \lambda_{o t}$ which can be then directly plugged into (??) and (??) $)_{2}$. Figure ?? shows the trend for the already selected different values of $j_{m}(50,100,200)$. As expected, when $j_{m}$ takes the largest value, the difference between the Gent and the neo-Hookean curve is smaller. In all cases, the difference in curvature prediction is significative only along the descending branch of the curve which eventually reaches zero curvature with a constant slope as Gent model allows for the existence of an ultimate deformation defined by the equation (??) and depending on $j_{m}$.

\subsubsection{Slope of the linear decaying branch close to the ultimate deformation}

Thanks to the availability of a closed form solution for the curvature of the bilayer beam, we can quantify the constant slope at which $\kappa h$ intersects the $\lambda_{o b}$ axis in Figure ??. To this end (??) is rewritten as

$0=\frac{g_{s}}{\lambda_{o s}} \frac{\lambda_{o, m}^{2}-1}{\lambda_{o, m}^{2}-\lambda_{o s}^{2}}+\frac{f\left(\lambda_{o s}\right)}{\lambda_{o s}^{6}}$

where $g_{s}$ is, with the addition of subscript $s=t, b$, the same non dimensional shear modulus defined in $(? ?)_{2}$.
Expanding (??) in series for $\lambda_{o s}$ in the neighbourhood of $\lambda_{o, m}$ and keeping only the leading order term of each addend one gets

$0=\frac{g_{s}}{2 \lambda_{o, m}} \frac{\lambda_{o, m}^{2}-1}{\lambda_{o, m}-\lambda_{o s}}+\frac{f\left(\lambda_{o, m}\right)}{\lambda_{o, m}^{6}}$,

where a $\left(\lambda_{o, m}-\lambda_{o s}\right)^{0}$ order term in the first addend of (??) was discarded after having seen that for $\lambda_{o s}$ sufficiently close to $\lambda_{o, m}$, it was uninfluential as far as the $g_{s}$ vs $\left(\lambda_{o, m}-\lambda_{o s}\right)$ relationship went. From (??) we see that $\left(\lambda_{o, m}-\lambda_{o s}\right) / g_{s}$ is a constant independent of $g_{s}$ and $\lambda_{o s}$. We notice again here that we have chosen the value of $\lambda_{o, m}$ to be the same in the top and bottom layers. In this way, given that $g_{t}=\alpha g_{b}$, we observe that $\left(\lambda_{o, m}-\lambda_{o t}\right)=\alpha\left(\lambda_{o, m}-\lambda_{o b}\right)$ and we can approximate $\Gamma$ as

$\Gamma=\frac{\lambda_{o, m}-\left(\lambda_{o, m}-\lambda_{o b}\right)}{\lambda_{o, m}-\alpha\left(\lambda_{o, m}-\lambda_{o b}\right)} \simeq 1-(1-\alpha)\left(1-\frac{\lambda_{o b}}{\lambda_{o, m}}\right)$

Now the asymptotic expression for $\Gamma$ (??) is plugged into the expression of the curvature obtained by substitution of (??) into (??) $)_{2}$. The leading order term of the series expansion of the resulting expression for $\lambda_{o b} \rightarrow \lambda_{o, m}$ yields the asymptotic $\kappa h$ vs $\lambda_{o b}$ relationship close to the ultimate deformation $\lambda_{o, m}$

$\kappa h \simeq \frac{6 \alpha(1-\alpha) \beta(1-\beta)}{(1-\alpha)\left((1-\beta)^{4}-\alpha \beta^{4}\right)+\alpha} \frac{\lambda_{o, m}-\lambda_{o b}}{\lambda_{o, m}^{2}}$.

Linear expression (??) is plotted in Figure ?? using dashed lines in order to appraise the accuracy of the approximation. In passing, we note that for fixed values of $\alpha$ and $\beta$, as occurs in Figure ??, the asymptotic slope in (??) is inversely proportional to $\lambda_{o, m}^{2}$ which in turn is, given (??), proportional to $j_{m}$. In this way, when $j_{m}$ is reduced by one half in Figure ?? from 100 to 50, the value of the asymptotic slope approximately doubles as can be schematically inferred from the plot.

\section{Numerical experiments via the fully coupled stress-diffusion model of bilayer gel beams}

With the aim to verify the non-monotonic pattern of the curvature vs free-swelling stretch curves shown in figure ??, we planned a set of numerical tests, based on the nonlinear three-dimensional stress-diffusion model shortly summed up in Section ??. As the Flory-Rehner free energy is not defined at dry state, we fixed as reference configuration of the bilayer gel beam an almost dry straight configuration corresponding to swelling ratios of the two components around 1.001, with a 
solvent bath chemical potential $\mu_{o}<0$, and compatible with the Young's moduli of the two homogeneous beam-like parts according to the free-swelling equilibrium equation (??). Moreover, we selected a constitutive prescription for the solvent flux (see ?) which satisfies the reduced dissipation inequality (??), as $\mathbf{h}=$ $-\mathbf{M}\left(\mathbf{F}_{d}, c_{d}\right) \nabla \mu$,

$\left.\mathbf{M}\left(\mathbf{F}_{d}, c_{d}\right)=c_{d} D / R T\left(\mathbf{F}_{d}^{T} \mathbf{F}_{d}\right)^{-1}\left([\mathbf{M}]=\mathrm{mol}^{2} / \mathrm{s} \mathrm{m} \mathrm{J}\right]\right)$ being the isotropic mobility tensor and $D\left([D]=\mathrm{m}^{2} / \mathrm{s}\right)$ the diffusivity.

The beam was constrained so as to allow the freeswelling change in shape; hence, because of the symmetry of the problem, we hampered the longitudinal displacement over the entire middle cross section, the out-of-plane displacement along the middle line in the middle cross section, and the transverse displacement at the bottom point of this line. The remaining boundary was assumed to be traction-free $(\mathbf{t}=\mathbf{0})$. We allowed the sample to freely swell in a solvent bath whose chemical potential $\mu_{e}$ was controlled and set to 0 .

We implemented and numerically solved the model through the finite element software COMSOL Multiphysics, recasting all the equations in a weak form. We refer to (???) for details, and only discuss here the boundary condition corresponding to bath conditions. The boundary condition (?? $)_{2}$ that, through equation $(? ?)_{2}$, relates the solvent concentration $c_{d}$ to the chemical potential $\mu_{e x t}$ allows to control the solvent concentration $c_{d}$ at the boundary through the chemical potential $\mu_{e x t}$ and is a highly nonlinear equation in $c_{d}$. It is enforced in weak form in terms of an additional state variable $c_{s}$, defined only on the boundary of the bilayer gel beam, representing the solvent concentration at boundary:

$\int_{\partial \mathcal{B}_{d}}\left(\mu_{c}\left(c_{s}\right)+p \Omega-\mu_{\mathrm{ext}}\right) \cdot \tilde{c}_{s}=0$.

Once (??) is solved for $c_{s}$, with $\mu_{\text {ext }}$ as an input, we set the pointwise constraint $c_{d}=c_{s}$ as boundary condition for the balance of solvent at any time on $\partial_{c} \mathcal{B}$. To this extent, the weak equation (??) may be viewed as resembling the idea of a surface physics, that is, a physics defined and solved only on the surface, and coupled to the bulk physics through the pointwise constraint. However, consideration of a complete physical surface over the boundary of the body would require the introduction of a surface concentration field (whose dimensions are $\left.\left[\mathrm{mol} / \mathrm{m}^{2}\right]\right)$ different from $c_{s}$, which is just the restriction of the bulk concentration $c_{d}$ to the boundary (see ? for more details).

Fixed $\beta=1 / 2, l=10 h, h / b=2$, and $b=10^{-3} \mathrm{~m}$, the analyses were performed for $\alpha=1 / 2,1 / 3,1 / 4$, according to the scheme shown in figure ??: given the

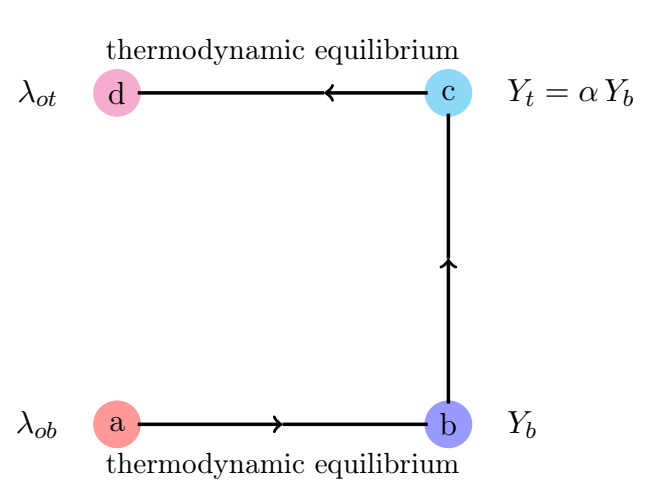

Figure 4 Fixed $\alpha$ and the swelling ratio $\lambda_{o b}$ of the bottom layer, through the thermodynamic equilibrium equation (??) the corresponding Young's moduli $Y_{b}$ and $Y_{t}=\alpha Y_{b}$ are evaluated; equation (??) also delivers the swelling ratio $\lambda_{o t}$ corresponding to $Y_{t}$.

swelling ratio $\lambda_{o b}$ of the bottom layer, through the thermodynamic equilibrium equation (??) the corresponding Young's modulus $Y_{b}$ was evaluated; being $\alpha$ fixed, the Young's modulus of the top layer was easily obtained and, again through equation (??), the corresponding swelling ratio $\lambda_{o t}$ was computed. For any values of the parameters, a uniform bending solution was obtained, corresponding to a plane bending; all solutions delivered a uniform beam axis curvature $\kappa$ which was evaluated at a distance equal to $l / 4$ from the end of the beam as

$\kappa=\frac{w^{\prime \prime}\left(1+u^{\prime}\right)-w^{\prime} u^{\prime \prime}}{\left(\left(1+u^{\prime}\right)^{2}+w^{\prime 2}\right)^{3 / 2}}$,

$u$ and $w$ being the longitudinal and transverse displacement fields at the beam axis, and a prime denoting derivative with respect to the axis coordinate. Numerical results are shown in figure ?? as dots, with colour code corresponding to the ratio $\alpha$; they confirm the hardening of the bilayer gel beam, as the layers are chosen softer and softer and $\alpha$ is held constant. Figure ?? also shows that the structural model here discussed increasingly overestimates beam curvature as $\alpha$ decreases. However, the overall agreement between numerical and analytical results, corresponding respectively to a fully three-dimensional stress diffusion model and to the approximated structural model, is very good. It is worth noting that the agreement holds when $h / b>1$, that is, for aspect ratios of the bilayer strip which result in an overall beam behaviour.

\section{The linear regime}

We now further generalize our model and consider $\lambda_{\text {os }}$ to be homogeneous, isotropic distortions, i.e. linear dilations, which are not necessarily due to swelling as in gels 


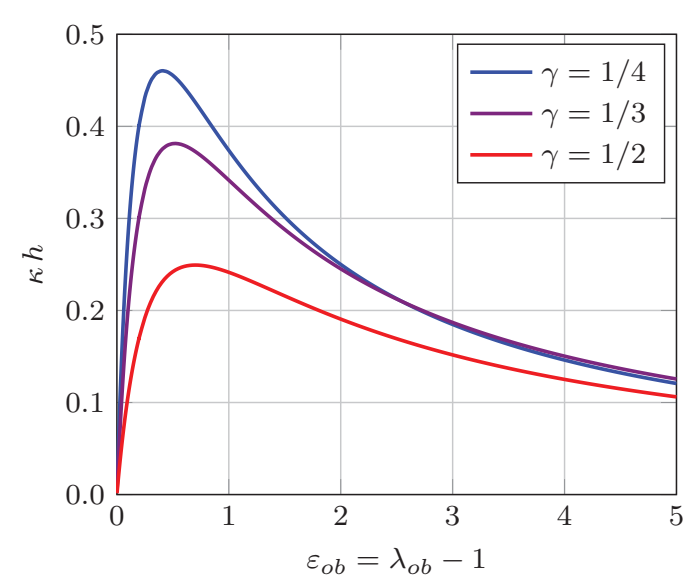

Figure 5 Curvature times thickness $\kappa h$ versus the swelling stretch of the bottom layer $\lambda_{o b}$ for $\alpha=1 / 3, \beta=1 / 2$ and $\gamma=1 / 4,1 / 3,1 / 2$.

but may have another, unspecified, nature, e.g. thermal. To relate the present model to the small strain setting assumed in (?), we introduce small distortions

$\varepsilon_{o s}=\lambda_{o s}-1, \quad \varepsilon_{o b}=\gamma \varepsilon_{o t}$,

and their ratio, $0 \leq \gamma \leq 1$. The ratio $\Gamma$ can be computed as a function of $\varepsilon_{o b}$ and $\gamma$ as

$\Gamma=\frac{\lambda_{o b}}{\lambda_{o t}}=\frac{1+\varepsilon_{o b}}{1+\varepsilon_{o b} / \gamma}$.

Longitudinal stretch $\Lambda_{0}$ and curvature $\kappa$ are recovered substituting (??) into equations (??)

$\Lambda_{0}=X_{0}\left(1+\varepsilon_{o b}\right)$ and $\kappa h=X_{1} X_{0}^{-2}\left(1+\varepsilon_{o b}\right)^{-1}$.

Substitution of (??) into (??) and (??) provides a generalization of Timoshenko's formula for finite strain applications. The resulting curvature vs. $\varepsilon_{o b}$ plot is shown in Figure ?? for $\alpha=1 / 3, \beta=1 / 2$ and $\gamma=1 / 4,1 / 3,1 / 2$. Two distinct curvature regimes are obtained expanding $\kappa h$ in series in the neighborhood of specific values of $\varepsilon_{o b}$. For $\varepsilon_{o b} \rightarrow 0$, the curvature is linear in $\varepsilon_{o b}$

$\kappa h=\frac{6 \alpha \beta(1-\beta)(1-\gamma)}{\gamma\left((1-\alpha)(1-\beta)^{4}+\alpha-\alpha(1-\alpha) \beta^{4}\right)} \varepsilon_{o b}$,

and the expression coincides with Timoshenko's once his parameters $m=\beta /(1-\beta)$ and $n=\alpha$ are substituted. For $\varepsilon_{o b} \rightarrow \infty$, the curvature is linear in $1 / \varepsilon_{o b}$

$\kappa h=\varepsilon_{o b}^{-1} \cdot 6 \alpha \beta(1-\beta) \gamma^{2}(1-\gamma)$.

. $\left(\alpha^{2} \beta^{4}+2 \alpha \beta(2-(1-\beta) \beta)(1-\beta) \gamma+(1-\beta)^{4} \gamma^{2}\right)$.

$\cdot\left(\alpha^{2} \beta^{4}+(1-\beta)^{4} \gamma^{2}(\gamma-\alpha)+\right.$

$$
\left.+\alpha \beta\left(1-\beta^{3}\right) \gamma+\alpha(1-\beta) \gamma^{2}\right)^{-2} .
$$

At this stage, we can attempt a simpler geometric explanation for the two regimes observed. Looking at equation (??), we can point out two main characteristics of the proposed bilayer beam model: 1) bending is multiplicatively superimposed to an expansion $\Lambda_{0}$ and 2) stretch is linear in the thickness coordinate $x_{3}$. Consistently with the above assumptions, we can compute the curvature as

$\kappa=\frac{\lambda_{o t}-\lambda_{o b}}{\Lambda_{0}} \cdot \frac{1}{\Lambda_{0} h / 2}$

where we schematically assign $\lambda \simeq \lambda_{o t}$ to the top beam and $\lambda \simeq \lambda_{o b}$ to the bottom one and where $\Lambda_{0} h / 2$ is the distance between the mean lines of the two beams. At this point we can distinguish between two regimes. For very large $\lambda_{o b}$, we have that $\Gamma$ is almost constant and therefore

$\lambda_{o t}=\frac{\lambda_{o b}}{\Gamma} \propto \lambda_{o b} \quad$ and $\quad \Lambda_{0} \simeq \frac{\beta \lambda_{o t}+(1-\beta) \lambda_{o b}}{2} \propto \lambda_{o b}$

whence one infers that $\kappa h$ in (??) is proportional to $1 / \lambda_{o b}$ for large values of $\lambda_{o b}$. For small strains, (??) holds with $\varepsilon_{o s}<<1$ and $\Lambda_{0}^{2} \simeq 1$ from which we can conclude that $\kappa h$ in (??) is proportional to $\varepsilon_{o b}$.

\section{Conclusions and future directions}

We have used an existing model for the evaluation of stretching and curvature of bilayer gel beams (?) to investigate and explain in detail an apparent paradox consisting in a decrease in curvature for softer layers at large strains. The model is simplified in that it neglects the chemo-mechanical coupling, but in this way it is applicable to general distorsions such as thermal ones etc... Though extensive tests have not been carried out yet, the model seems to perform better for aspect ratios closer to beams $(h / b>1)$ than to ribbons $(h / b<1)$. However, in the given aspect ratio range and for a fairly wide parameter set, results are accurate when compared to fully-coupled three-dimensional numerical analyses. In addition to the explanation of the unexpected hardening, the paper provides (1) a validation with numerical analyses, (2) a confirmation of the phenomenon also in the case of stiffening effects at large strains accounted for by Gent's model (??), (3) a generalization at large strain for the well-known Timoshenko's formula (?) for the thermally induced bending of bilayer beams, (4) and several closed-form analytical results useful for prediction and design which can be extended using the template recurrently used in the paper.

We didn't analyze extremal situations such as very thin gel layers glued over stiffer elastomeric layers; as it was noted in (?) through the fully three-dimensional stress diffusion model, in these situations wrinkling appears on the top surface of the gel layer, in addition 
to overall bending. We plan to investigate the surface wrinkling arising under these circumstances starting from the model set in (?) and looking for solutions allowing for creases and wrinkling of the top gel layer, following the path described in (?).

Acknowledgements E.P. acknowledges the National Group of Mathematical Physics (GNFM-INdAM) for support (Young Researcher Project).

\section{References}

Agostiniani V, De Simone A (2017) Dimension reduction via Gamma-convergence for soft active materials. Meccanica, this issue.

Armon S, Efrati E, Kupferman R, Sharon E (2011) Geometry and mechanics in the opening of chiral seed pods. Science 333(6050):1726-1730, DOI $10.1126 /$ science. 1203874

Baek K, Jeong JH, Shkumatov A, Bashir R, Kong H (2013) In situ self-folding assembly of a multi-walled hydrogel tube for uniaxial sustained molecular release. Advanced Materials 25(39):5568-5573, DOI 10. 1002/adma.201300951, URL http://dx.doi.org/ 10.1002/adma. 201300951

Cai S (2015) Bending a beam by a generalized ideal elastomeric gel. Proceedings of the Royal Society of London A: Mathematical, Physical and Engineering Sciences 471(2175), DOI 10.1098/rspa.2014.0919, URL http://rspa.royalsocietypublishing. org/content/471/2175/20140919, http: //rspa.royalsocietypublishing.org/content/ 471/2175/20140919.full.pdf

De Tommasi D, Puglisi G, Zurlo G (2013) Inhomogeneous deformations and pull-in instability in electroactive polymeric films. International Journal of Non-Linear Mechanics 57:123 - 129, DOI http: //dx.doi.org/10.1016/j.ijnonlinmec.2013.06.008,

URL http://www.sciencedirect.com/science/ article/pii/S0020746213001236

Drozdov A, deClaville Christiansen J (2016) Swelling-induced bending of bilayer gel beams. Composite Structures pp -, DOI http://dx.doi.org/10.1016/j.compstruct.2016.06.076, URL http://www.sciencedirect.com/science/ article/pii/S0263822316310716

Erb RM, Sander JS, Grisch R, Studart A (2013) Selfshaping composites with programmable bioinspired microstructures. Nat Commun 4(10):1712, DOI 10. 1038/ncomms2666

Fang Y, Pence TJ, Tan X (2008) Nonlinear elastic modeling of differential expansion in trilayer conjugated polymer actuators
Flory PJ, Rehner J (1943a) Statistical mechanics of cross-linked polymer networks i. rubberlike elasticity. J Chem Phys 11(11):512-520

Flory PJ, Rehner J (1943b) Statistical mechanics of cross-linked polymer networks ii. swelling. J Chem Phys 11(11):521-526

Gent AN (1996) A new constitutive relation for rubber. Rubber Chemistry and Technology 69(1):5961, DOI 10.5254/1.3538357, URL http://dx.doi. org/10.5254/1.3538357, http://dx.doi.org/10. $5254 / 1.3538357$

Hu Z, Zhang X, Li Y (1995) Synthesis and application of modulated polymer gels. Science 269(5223):525527, DOI 10.1126/science.269.5223.525, URL http://science.sciencemag.org/content/269/ 5223/525, http://science.sciencemag.org/ content/269/5223/525.full.pdf

Lucantonio A, Nardinocchi P, Teresi L (2013) Transient analysis of swelling-induced large deformations in polymer gels. Journal of the Mechanics and Physics of Solids 61(1):205 - 218, DOI http://dx.doi.org/10.1016/j.jmps.2012.07.010, URL http://www.sciencedirect.com/science/ article/pii/S0022509612001548

Lucantonio A, Nardinocchi P, Pezzulla M (2014) Swelling-induced and controlled curving in layered gel beams 470(2171), DOI 10.1098/rspa.2014.0467

Lucantonio A, Teresi L, DeSimone A (2016) Continuum theory of swelling material surfaces with applications to thermo-responsive gel membranes and surface mass transport. Journal of the Mechanics and Physics of Solids 89:96 - 109, DOI http://dx.doi.org/10.1016/j.jmps.2016.02.001, URL http://www.sciencedirect.com/science/ article/pii/S0022509616300746

Morimoto T, Ashida F (2015) Temperature-responsive bending of a bilayer gel. International Journal of Solids and Structures 5657:20 - 28, DOI http://dx.doi.org/10.1016/j.ijsolstr.2014.12.009, URL http://www.sciencedirect.com/science/ article/pii/S0020768314004776

Nardinocchi P, Puntel E (2016) Finite bending solutions for layered gel beams. International Journal of Solids and Structures 90:228 - 235, DOI http://dx.doi.org/10.1016/j.ijsolstr.2016.02.026, URL http://www.sciencedirect.com/science/ article/pii/S0020768316000883

Nardinocchi P, Pezzulla M, Teresi L (2015) Steady and transient analysis of anisotropic swelling in fibered gels. Journal of Applied Physics 118(24):244904, DOI http://dx.doi.org/10.1063/1.4938737, URL http://scitation.aip.org/content/aip/ journal/jap/118/24/10.1063/1.4938737 
Puglisi G, Saccomandi G (2015) The gent model for rubber-like materials: An appraisal for an ingenious and simple idea. International Journal of Non-Linear Mechanics 68:17 - 24, DOI http: //dx.doi.org/10.1016/j.ijnonlinmec.2014.05.007,

URL http://www.sciencedirect.com/science/ article/pii/S0020746214001097, mechanics of Rubber - in Memory of Alan Gent

Timoshenko S (1925) Analysis of bimetal thermostats. JOSA 11:233-255 